\title{
CORRECTION
}

\section{Correction to: The Human Right to Science and the Regulation of Human Germline Engineering by Boggio A, Knoppers BM, and Romano CPR. CRISPR J 2019;2:134-142. DOI: 10.1089/crispr.2018.0053}

In the June 2019 issue of The CRISPR Journal (vol. 2, no. 3; 134-142) the article entitled "The Human Right to Science and the Regulation of Human Germline Engineering" by Boggio et al. requires correction.

On page 137, the authors listed China among the countries in which "embryo research is not prohibited, but scientists can only use embryos that were created for reproductive purposes, and that will not be used (so-called in vitro fertilization [IVF] supernumerary embryos)." China should have appeared in the following sentence among the "handful of countries (Belgium, Israel, Singapore, Sweden, the United Kingdom, and several states of the United States), [in which] scientists can create embryos for research purposes."

The paragraph originally read:

"In Australia, Canada, China, France, Japan, South Korea, Mexico, the Netherlands, and Spain, embryo research is not prohibited, but scientists can only use embryos that were created for reproductive purposes and that will not be used (so-called in vitro fertilization [IVF] supernumerary embryos). In a handful of countries (Belgium, Israel, Singapore, Sweden, the United Kingdom, and several states of the United States), scientists can create embryos for research purposes."

The paragraph should now read:

In Australia, Canada, France, Japan, South Korea, Mexico, the Netherlands, and Spain, embryo research is not prohibited, but scientists can only use embryos that were created for reproductive purposes and that will not be used (so-called in vitro fertilization [IVF] supernumerary embryos). In a handful of countries (Belgium, China, Israel, Singapore, Sweden, the United Kingdom, and several states of the United States), scientists can create embryos for research purposes.

The online version of the article has been corrected to reflect this. The authors apologize for this error. 